\title{
Generalized Eigen-analysis of SSO Based on Novel Structure-preserving LDAE Model
}

\author{
Chang Yu, and Yixin Ni, Senior Member, IEEE
}

\begin{abstract}
A novel structure-preserving linearized differential and algebraic equations (LDAE) model for small signal eigenanalysis of power system SSO is proposed in this paper. LDAE models of various power system components are formed first. Modularized establishment of entire system model is then conducted quickly according to the network topology. Generalized eigenvalue \& eigenvector calculation, and eigenvalue sensitivity analysis based on the LDAE model are applied thereafter. The effectiveness of the proposed LDAE model based SSO analysis approach is verified through computer test results. The LDAE-based SSO study model and the corresponding generalized eigen-analysis approach pave the way for power system SSO study with HVDC transmission and/or FACTS devices without eliminating any algebraic variables.
\end{abstract}

Index Terms-- SSO, Structure-preserving, LDAE, Generalized eigenvalue sensitivity analysis

\section{INTRODUCTION}

$S^{u}$ ubsynchronous Oscillation (SSO) is a complex stability issue in power systems and there have been a lot of research outputs on SSO in recent decades ${ }^{[1]}$. The preliminary studies on SSO concerned mainly about induction generator effect induced by series compensation capacitor. In early 1970s, series compensation stimulated electromechanical torsional interaction, which caused severe torsional oscillations and damage of turbine-generator shafts, attracted great attention and research work. This led to a new period of SSO study. In late 1970's, HVDC transmission system caused SSO was first observed and brought about in-depth studies on device-dependent SSO. The application of FACTS technology has promoted the study on device-dependent SSO even more significantly ${ }^{[2 \sim 4]}$.

In order to study power system SSO, various methods are used ${ }^{[5,6]}$. Among them time simulation is the most direct way, in which EMT models of power system elements with multimass spring shaft effects and step-by-step numerical integration methods are used to get time-evolution of various variables in SSO. A distinct advantage of time simulation method is that it allows detailed modeling of power system elements with system nonlinearity included. Therefore it is very useful for the study of transient torque amplifying effect

Chang Yu is with the EEE Department, Hong Kong University, Hong Kong. (e-mail: changyu@eee.hku.hk)

Yixin Ni is with the EEE Department, Hong Kong University, Hong Kong. (e-mail:yxni@eee.hku.hk) and the examination of SSO damping control performance under large disturbances. However, using EMT models for SSO study is time consuming for its extremely small time step especially when there are HVDC transmission and/or FACTS devices in the studied power system. Besides, time simulation is poor in physical transparency and difficult to reveal SSO principles. Small signal stability study is another method widely used for SSO study, where the power system model is linearized around system operation point and converted to a standard form of $\dot{\boldsymbol{x}}=\boldsymbol{A}_{S} \boldsymbol{x}$ ( $\boldsymbol{A}_{S}$ is the system state-space matrix). Eigen-analysis is then conducted to get deep in-sight of system SSO characteristics. Eigen-analysis is an accurate method which can examine the effects of system control and parameters on damping the torsional modal oscillation. With the help of mature linear control theories, advanced SSO damping control can be realized. However, in a real power system with HVDC transmission and/or FACTS devices, it is time-consuming in deriving system matrix $\boldsymbol{A}_{S}$ with all the algebraic variables eliminated. Moreover, after reduction of algebraic variables, it may be inconvenient to conduct certain eigenvalue sensitivity analysis based on $\boldsymbol{A}_{S}$. These issues in conventional eigen-analysis will be addressed in this paper. In recent years frequency-scanning based methods become computationally attractive because they can provide system damping information over the entire subsynchronous frequency bands ${ }^{[7]}$, although they are not so precise as eigenanalysis method. Among them the complex torque coefficient method (or say torque per unit speed method) is most suitable to real power system SSO analysis with the capability of showing clearly the system electric damping effects over SSO frequency band. The method suggested in this paper can be extended to realize the frequency-scanning function and get torque per unit speed information.

In this paper, a novel structure-preserving linearized differential and algebraic equations (LDAE) model for small signal eigen-analysis of SSO is proposed. In the LDAE approach, the individual power system components described by their LDAEs are acting as building blocks, and the system model can be established quickly and easily according to the network topology. This method is very suitable for SSO study of power systems with HVDC transmission and/or FACTS devices without the need of eliminating algebraic variables. Generalized eigen-analysis technique ${ }^{[10]}$ can be directly applied to the LDAE model. The results can provide useful information of observability and controllability of algebraic 
variables on individual SSO modes. Since the LDAE model preserves all algebraic variables and system structure, it is also very convenient to conduct eigenvalue sensitivity analysis based on the extended system matrix and generalized right \& left eigenvectors. Furthermore, from LDAE model the complex torque coefficient can be easily calculated, which will be discussed in detail in another paper. The effectiveness of the proposed LDAE model based SSO analysis is verified with computer test results of IEEE First Benchmark Model. The LDAE-based SSO study model and its corresponding analysis approach pave the way to include HVDC transmission and FACTS device models in SSO study and get deep insight about their effects on SSO.

The paper is arranged as follows. The structure-preserving LDAE model for SSO is derived in section 2. The LDAEmodel-based eigenvalue \& eigenvector computation, eigenvalue sensitivity analysis for SSO are presented in section 3, with computer test results given in section 4 and conclusions drawn in section 5 .

\section{STRUCTURE-PRESERVING LDAE MODEL FOR SSO STUDY}

Power systems can be described by a set of nonlinear differential and algebraic equations (DAE):

$$
\left\{\begin{array}{l}
\dot{x}=f(x, y) \\
0=g(x, y)
\end{array}\right.
$$

where $\boldsymbol{x}$ : the vector of state variables; $\boldsymbol{y}$ : the vector of algebraic variables

Linearizing the above equations around the operation point we have:

$$
\left\{\begin{array}{l}
\Delta \dot{x}=A \Delta x+B \Delta y \\
0=C \Delta x+D \Delta y
\end{array}\right.
$$

where $\boldsymbol{A}=\frac{\partial f}{\partial x}, \boldsymbol{B}=\frac{\partial f}{\partial y}, \boldsymbol{C}=\frac{\partial g}{\partial x}, D=\frac{\partial g}{\partial y}$ calculated at the equilibrium point; $\Delta$ : an operator to represent perturbed values.

From (1), we can eliminate all algebraic variables $\boldsymbol{y}$ if $\boldsymbol{D}^{-1}$ exists, which leads to linearized ordinary differential equations (LODE) model in state space:

$$
\Delta \dot{x}=A_{S} \Delta x
$$

where system matrix $\boldsymbol{A}_{S}=\boldsymbol{A}-\boldsymbol{B} \boldsymbol{D}^{-1} \boldsymbol{C}$. Equation (2) is widely used for conventional eigen-analysis as mentioned above.

However, to eliminate all the algebraic variables in real power systems with HVDC transmission or/and FACTS devices might be time consuming. Moreover the item of " $\boldsymbol{B} \boldsymbol{D}^{-1} \boldsymbol{C}$ " makes $\boldsymbol{A}_{S}$ unable to be expressed by system parameters explicitly, which, under certain circumstances, increases the difficulty in eigenvalue sensitivity analysis. In this paper, the system sub-matrices $\boldsymbol{A}, \boldsymbol{B}, \boldsymbol{C}, \boldsymbol{D}$ will be kept, and eigen-analysis for SSO study will be based on the system LDAE model (1) directly. The details of LDAE formation procedure for SSO study are given below.

\section{A. Power System Component Model}

Power system component models for SSO study can be found in [9]. After linearization of component models at operation point, we can get LDAE models with algebraic variables preserved for various components as follows.

1). The Synchronous Machine Model

a) Spring-mass Shaft System

The shaft of a turbine generator can be expressed as a sixmass spring system, and its LDAE model is ( $\boldsymbol{X}$ : state variables; $\boldsymbol{Y}$ : algebraic variables):

$$
\dot{\boldsymbol{X}}_{S}=\boldsymbol{A}_{S S} \boldsymbol{X}_{S}+\boldsymbol{B}_{S T e} \boldsymbol{Y}_{T e}
$$

where $\boldsymbol{X}_{S}=\left(\Delta \delta_{1} \ldots \Delta \delta_{6}, \Delta \omega_{1} \ldots \Delta \omega_{6}\right)^{\mathrm{T}}$ : angle and speed increments of the six-mass shaft; $\boldsymbol{Y}_{T e}=\Delta T_{\mathrm{e}}$ : electromagnetic torque of generator mass (For simplicity, the speed governor's dynamics is neglected), which can be expressed as a function of generator winding currents and flux linkages in $d-q$ coordinates (in LDAE format):

$$
\boldsymbol{0}=\boldsymbol{C}_{G T e} \boldsymbol{X}_{G}+\boldsymbol{D}_{i T e} \boldsymbol{Y}_{i}+\boldsymbol{D}_{T e i} \boldsymbol{Y}_{T e}
$$

where $\boldsymbol{X}_{G}=\left(\Delta \psi_{d}, \Delta \psi_{q}, \Delta \psi_{f}, \Delta \psi_{D}, \Delta \psi_{g}, \Delta \psi_{Q}\right)^{\mathrm{T}}$ : generator winding flux linkages in $d$ - $q$ coordinates assuming a generator has six windings; and $\boldsymbol{Y}_{i}=\left(\Delta i_{d}, \Delta i_{q}, \Delta i_{f}, \Delta i_{D}, \Delta i_{g}, \Delta i_{Q}\right)^{\mathrm{T}}$ : generator winding currents in $d$ - $q$ coordinates.

To keep $\boldsymbol{Y}_{T e}$ and (4) explicitly in system model is good for future frequency-scanning calculation.

b) Electromagnetic Circuit

The winding voltage equations in $d-q$ coordinates in LDAE format are:

$$
\dot{\boldsymbol{X}}_{G}=\boldsymbol{A}_{G S} \boldsymbol{X}_{S}+\boldsymbol{A}_{G G} \boldsymbol{X}_{G}+\boldsymbol{B}_{G i} \boldsymbol{Y}_{i}+\boldsymbol{B}_{G u} \boldsymbol{Y}_{u}
$$

where $\boldsymbol{Y}_{u}=\left(\Delta u_{d}, \Delta u_{q}, \Delta u_{f}\right)^{\mathrm{T}}$ : generator winding voltages in $d$ $q$ coordinates with $\Delta u_{D}=\Delta u_{g}=\Delta u_{Q} \equiv 0$.

The linearized flux linkage equations (in $d$ - $q$ coordinates) are:

$$
\boldsymbol{O}=\boldsymbol{C}_{G i} \boldsymbol{X}_{G}+\boldsymbol{D}_{G i} \boldsymbol{Y}_{i}
$$

\section{2). Excitation System Model}

In this paper a $1^{\text {st }}$ order excitation system is considered for simplicity, and its LDAE model is:

$$
\begin{gathered}
\dot{\boldsymbol{X}}_{E}=\boldsymbol{A}_{E E} \boldsymbol{X}_{E}+\boldsymbol{B}_{E u} \boldsymbol{Y}_{u} \\
\boldsymbol{O}=\boldsymbol{C}_{E u} \boldsymbol{X}_{E}+\boldsymbol{D}_{E u} \boldsymbol{Y}_{u}
\end{gathered}
$$

where $\boldsymbol{X}_{E}=\Delta E_{f}$ : output voltage of excitation system. Relation $\Delta E_{f}=\frac{X_{a d}}{r_{f}} \Delta u_{f}$ is expressed as (8).

\section{3). Transmission Network Component Model}

All ac network components adopt EMT models in $x-y$ synchronous coordinates.

a) Model for AC Transmission Lines without Series Capacitor Compensation (line type-A)

The $\pi$-equivalent circuit is used to describe ac transmission lines with line charging capacitance considered separately. Hence the LDAE model for ac line $l$ (i.e. an $R-L$ branch) from bus $i$ to bus $j$ without series capacitor compensation is:

$$
\dot{\boldsymbol{X}}_{A}=\boldsymbol{A}_{A A} \boldsymbol{X}_{A}+\boldsymbol{A}_{A U} \boldsymbol{X}_{U}
$$

where $\boldsymbol{X}_{A}=\left(\Delta i_{l x}, \Delta i_{l y}\right)^{\mathrm{T}}$ : line current; $\boldsymbol{X}_{U}=\left(\ldots \Delta u_{i x}, \Delta u_{i y} \ldots \Delta u_{j x}\right.$ , $\left.\Delta u_{j y} \ldots\right)^{\mathrm{T}}$ : all the bus voltages except the generator terminal 
buses. $\boldsymbol{X}_{U}$ are treated as state variables because of the existence of line charging capacitance.

b) Model for Special AC Transmission Lines with Series Capacitor Compensation (line type-B)

Similarly, we can derive the LDAE model for branch $k$ (i.e. an $R$ - $L-C$ branch) from bus $i$ to bus $j$ with series capacitor compensation:

$$
\left\{\begin{array}{l}
\dot{\boldsymbol{X}}_{B}=\boldsymbol{A}_{B B} \boldsymbol{X}_{B}+\boldsymbol{A}_{B C} \boldsymbol{X}_{C}+\boldsymbol{A}_{B U} \boldsymbol{X}_{U} \\
\dot{\boldsymbol{X}}_{C}=\boldsymbol{A}_{C B} \boldsymbol{X}_{B}+\boldsymbol{A}_{C C} \boldsymbol{X}_{C}
\end{array}\right.
$$

where $\boldsymbol{X}_{B}=\left(\Delta i_{k x}, \Delta i_{k y}\right)^{\mathrm{T}}$ : line current; $\boldsymbol{X}_{C}=\left(\Delta u_{C x}, \Delta u_{C y}\right)^{\mathrm{T}}$ : voltage drop on series compensation capacitor.

\section{c) Model for Transformer Branches}

If we only consider short circuit impedance of transformer, the transformer branch can be dealt with in the same way as type-A transmission lines. If transformer tap ratios should be included, then additional algebraic equations need to be added to describe the voltage and current relations between the primary and secondary windings of an ideal transformer. In this paper, only transformer short circuit impedance is considered in SSO study. The LDAE model for transformer branch is:

$$
\dot{\boldsymbol{X}}_{T}=\boldsymbol{A}_{T T} \boldsymbol{X}_{T}+\boldsymbol{A}_{T U} \boldsymbol{X}_{U}+\boldsymbol{B}_{T u}^{(x y)} \boldsymbol{Y}_{u}^{(x y)}
$$

where $\boldsymbol{X}_{T}=\left(\Delta i_{T x}, \Delta i_{T y}\right)^{\mathrm{T}}$ : transformer branch current; $\boldsymbol{Y}_{u}^{(x y)}=$ $\left(\Delta u_{x}{ }^{(G)}, \Delta u_{y}{ }^{(G)}\right)^{\mathrm{T}}$ : generator terminal bus voltage in $x-y$ synchronous coordinates. If a transformer is not connected to generator directly, then the corresponding third item on the right hand side of (11) is equal to 0 .

\section{d) Model for Equivalent Shunt Capacitance Branches}

For each bus, line charging capacitance and shunt compensation capacitance will be merged together as an equivalent capacitance. Its LDAE model will be:

$$
\dot{\boldsymbol{X}}_{U}=\boldsymbol{A}_{U U} \boldsymbol{X}_{U}+\boldsymbol{B}_{U i}^{(C)} \boldsymbol{Y}_{i}^{(C)}
$$

where $\boldsymbol{Y}_{i}^{(C)}=\left(\Delta i_{x}{ }^{(C)}, \Delta i_{y}{ }^{(C)}\right)^{\mathrm{T}}$ : shunt capacitance branch current.

\section{e) Model for Load Branches}

The load is modeled as linear impedance and its LDAE model is:

$$
\dot{\boldsymbol{X}}_{L}=\boldsymbol{A}_{L L} \boldsymbol{X}_{L}+\boldsymbol{A}_{L U} \boldsymbol{X}_{U}
$$

where $\boldsymbol{X}_{L}=\left(\Delta i_{x}{ }^{(L)}, \Delta i_{y}{ }^{(L)}\right)^{\mathrm{T}}$ : load branch current.

\section{B. The overall System LDAE Model for SSO Analysis}

Based on the component models described above, the overall system linearized DAE model for SSO analysis can be built up according to the network topology. Some key points will be emphasized here before constitution of system model.

(i) As shown in component LDAE models, we preserve the algebraic variables $\boldsymbol{Y}_{u}$ and $\boldsymbol{Y}_{i}$ (generator terminal voltages and injection currents in $d-q$ coordinates) and their companion variables $\boldsymbol{Y}_{u}{ }^{(x y)}$ and $\boldsymbol{Y}_{i}^{(x y)}=\left(\Delta i_{x}{ }^{(G)}, \Delta i_{y}{ }^{(G)}\right)^{\mathrm{T}}$ in $x-y$ synchronous coordinates. Hence, the algebraic equations for coordinates transformation between $d-q$ and $x-y$ can be directly included in system model as shown in (14):

$$
\left[\begin{array}{c}
\Delta f_{d} \\
\Delta f_{q}
\end{array}\right]=\left[\begin{array}{cc}
\sin \delta_{0} & -\cos \delta_{0} \\
\cos \delta_{0} & \sin \delta_{0}
\end{array}\right]\left[\begin{array}{c}
\Delta f_{x} \\
\Delta f_{y}
\end{array}\right]+\left[\begin{array}{c}
f_{q 0} \\
-f_{d 0}
\end{array}\right] \Delta \delta
$$

where $f$ can be $u$ or $i$ to denote generator terminal voltage or generator injection current respectively; $\delta$ : rotor angle of generator-mass which is the leading angle of its $q$-axis w.r.t. the synchronous coordinate $x$-axis.

Keeping (14) in DAE model is beneficial to fast interface of generator with ac network. Equation (14) for generator terminal voltages and injection currents coordinates transformation can be rewritten to:

$$
\left\{\begin{array}{l}
\boldsymbol{0}=\boldsymbol{C}_{S i} \boldsymbol{X}_{S}+\boldsymbol{D}_{i i} \boldsymbol{Y}_{i}+\boldsymbol{D}_{i i}^{(x y)} \boldsymbol{Y}_{i}^{(x y)} \\
\boldsymbol{0}=\boldsymbol{C}_{S u} \boldsymbol{X}_{S}+\boldsymbol{D}_{u u} \boldsymbol{Y}_{u}+\boldsymbol{D}_{u u}^{(x y)} \boldsymbol{Y}_{u}^{(x y)}
\end{array}\right.
$$

(ii) According to the network topology, the following bus-line incident matrixes can be obtained. $\boldsymbol{C}_{N A}, \boldsymbol{C}_{N B}, \boldsymbol{C}_{N T}, \boldsymbol{C}_{N L}, \boldsymbol{D}_{N G}$ and $\boldsymbol{D}_{N C}$ are the incident matrices of buses w.r.t. type-A lines, type-B lines, transformer branches, load branches, generator branches, and bus equivalent shunt capacitance branches respectively. Based on above incident matrixes, bus injection current balance equations based on $K C L$ will be:

$\boldsymbol{0}=\boldsymbol{C}_{N A} \boldsymbol{X}_{A}+\boldsymbol{C}_{N B} \boldsymbol{X}_{B}+\boldsymbol{C}_{N T} \boldsymbol{X}_{T}+\boldsymbol{C}_{N L} \boldsymbol{X}_{L}+\boldsymbol{D}_{N G} \boldsymbol{Y}_{i}^{(x y)}+\boldsymbol{D}_{N C} \boldsymbol{Y}_{i}^{(C)}$

Equations (3) (13), (15) and (16) constitute the entire system structure-preserving LDAE model for SSO study as shown in (17), where the algebraic equations include the generator electromagnetic torque computation equations; the generator winding flux linkage equations; the relation of $E_{f}$ and $u_{f}$; the coordinates transformation equations for generator currents and voltanges; and bus injection current balance equations.

A brief discussion on (17) is made as follows.

(i) Matrices $\boldsymbol{A}, \boldsymbol{B}, \boldsymbol{C}$, and $\boldsymbol{D}$ are extremely sparse. In $\boldsymbol{A}$ and $\boldsymbol{D}$, the non-zero block-matrices are mainly on the diagonal and most of them are also very sparse. Hence sparse matrix techniques can be use to conduct small signal eigen-analysis. The computation work can decrease noticeably, which is significant to SSO study of real power systems.

(ii) The structure-preserving formulation approach breaks down the modeling issue into two parts, one is elementmodeling part and another is network topology and integration part. Therefore, if we want to study the impacts of HVDC transmission and/or FACTS devices on SSO, we can just easily add their individual LDAE models to (17); and then modify the corresponding bus-line incident matrix to include their injection current effects to the bus injection current balance equations (16). The process is simple and direct.

(iii) The generalized eigenvalue and eigenvector calculation can be directly applied to the LDAE model. The corresponding generalized eigenvectors can provide the information of observability and controllability of both state and algebraic variables on a certain SSO mode. This is a significant feature of the suggested method, which can provide deep insight into system SSO characteristics and is beneficial to controller design for SSO damping.

(iv) The suggested LDAE model formulation can avoid sacrificing the physical structure transparency due to the elimination of algebraic variables in traditional LODE 
modeling. Since system parameters can be shown explicitly in the LDAE model, it is convenient to make eigenvalue

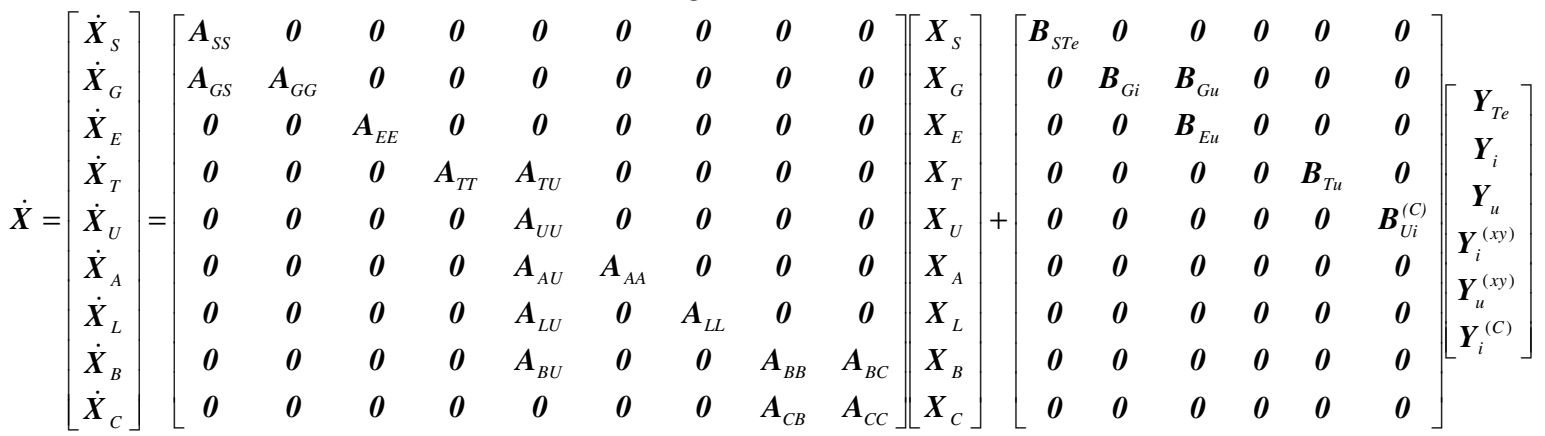

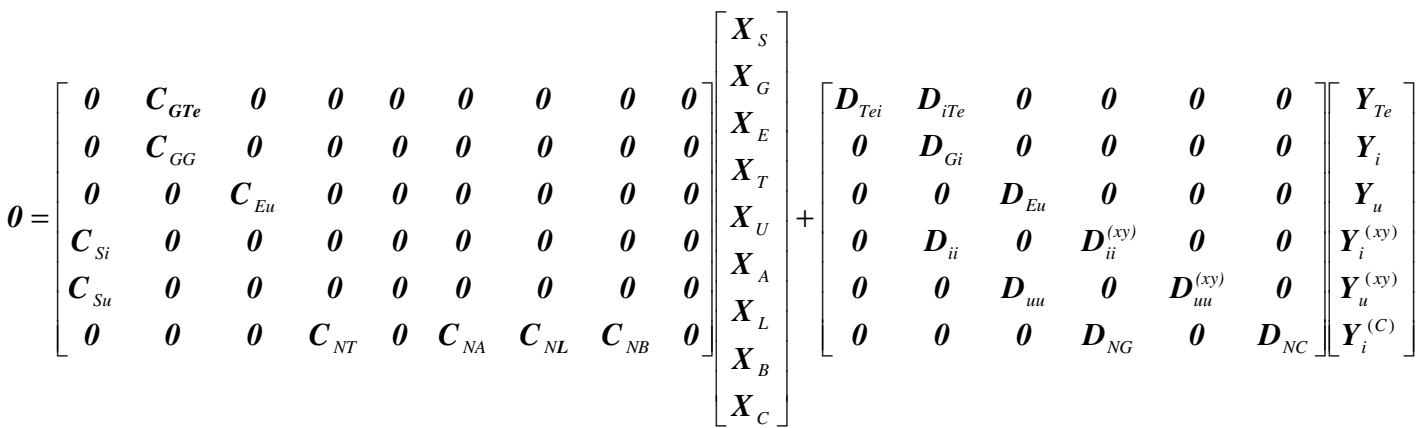

\section{EIGENVALUE \& EIGENVECTOR COMPUTATION, EIGENVALUE SENSITIVITY ANALYSIS BASED ON LDAE MODEL}

A. Eigenvalue and Eigenvector Computation Based on LDAE Model

LDAE model (17) can be written as:

$$
\left[\begin{array}{c}
\dot{X} \\
\boldsymbol{O}
\end{array}\right]=\left[\begin{array}{ll}
\boldsymbol{A} & \boldsymbol{B} \\
\boldsymbol{C} & \boldsymbol{D}
\end{array}\right]\left[\begin{array}{l}
\boldsymbol{X} \\
\boldsymbol{Y}
\end{array}\right]
$$

For the traditional eigen-analysis, we usually eliminate algebraic variables $\boldsymbol{Y}$ and get:

$$
\dot{\boldsymbol{X}}=\boldsymbol{A}_{S} \boldsymbol{X}
$$

where $\boldsymbol{A}_{S(n \times n)}=\boldsymbol{A}-\boldsymbol{B} \boldsymbol{D}^{-1} \boldsymbol{C}$ if $\boldsymbol{D}^{-1}$ exists.

Clearly, $\boldsymbol{A}_{S}$ might not be sparse. Its physical transparency may be lost due to the elimination of algebraic variables. Eigenvalue and eigenvector computation based on (19) is given in (20):

$$
\left\{\begin{array}{l}
\operatorname{det}\left(\lambda \boldsymbol{I}-\boldsymbol{A}_{S}\right)=0 \\
\lambda_{i} \boldsymbol{e}_{i}=\boldsymbol{A}_{S} \boldsymbol{e}_{\boldsymbol{i}}, \lambda_{i} \boldsymbol{f}_{i}=\boldsymbol{A}_{S}^{\mathrm{T}} \boldsymbol{f}_{i}
\end{array}\right.
$$

where $\boldsymbol{I}$ is identity matrix; $\boldsymbol{e}_{i}$ and $\boldsymbol{f}_{i}$ are the right and left eigenvectors with respect to the $i^{\text {th }}$ eigenvalue $\left(\lambda_{i}\right)$ of $\boldsymbol{A}_{S}$. The eigenvectors $\boldsymbol{e}_{i}$ and $\boldsymbol{f}_{i}$ can be normalized to satisfy $\boldsymbol{e}_{i}^{\mathrm{T}} \boldsymbol{f}_{i}=1$.

For LDAE model (18), we shall conduct generalized eigenvalue and eigenvector calculation using generalized Schur decomposition method ${ }^{[10]}$. For the suggested LDAE model (18), if we define $\boldsymbol{T}=\left[\begin{array}{l}\boldsymbol{X} \\ \boldsymbol{Y}\end{array}\right] ; \tilde{\boldsymbol{A}}=\left[\begin{array}{ll}\boldsymbol{A} & \boldsymbol{B} \\ \boldsymbol{C} & \boldsymbol{D}\end{array}\right]$ : extended system matrix; $\tilde{\boldsymbol{H}}=\left[\begin{array}{cc}\boldsymbol{I}_{n \times n} & \boldsymbol{0}_{n \times m} \\ \boldsymbol{0}_{m \times n} & \boldsymbol{0}_{m \times m}\end{array}\right]$ (where $n$ is the number of the state variables in $\boldsymbol{X}$ and $m$ is the number of the algebraic variables in $\boldsymbol{Y}$ ), we can rewrite (18) into:

$$
\tilde{\boldsymbol{H}} \dot{\boldsymbol{T}}=\tilde{\boldsymbol{A}} \boldsymbol{T}
$$

The solution of equation (21) is equivalent to solve the generalized eigenvalue problem (22):

$$
\lambda \tilde{H} \boldsymbol{T}=\tilde{A} T
$$

If $\tilde{\boldsymbol{H}}$ is nonsingular, we can convert the problem to a standard eigen-problem $\left(\tilde{\boldsymbol{H}}^{-1} \tilde{\boldsymbol{A}}\right) \boldsymbol{T}=\lambda \boldsymbol{T}$ to solve the generalized eigenvalues of (22). However, for our case, $\tilde{\boldsymbol{H}}$ is singular and $\widetilde{\boldsymbol{H}}^{-1}$ does not exist. An advanced eigenvalue computation technique, i.e. generalized Schur decomposition method ${ }^{[10]}$, is adopted, through which the inverse calculation of $\tilde{\boldsymbol{H}}$ can be avoided and the original system eigenvalues can be calculated efficiently. Hence this is an ideal method to solve the special generalized eigen-problem with rankdeficient $\tilde{\boldsymbol{H}}$.

According to generalized Schur decomposition for a pair of complex square matrices $(\tilde{\boldsymbol{H}}, \tilde{\boldsymbol{A}})$ of same dimension $(l \times l)$, we can find a pair of unitary matrices $\boldsymbol{Q}_{(l \times l)}$ and $\boldsymbol{Z}_{(l \times l)}\left(\boldsymbol{Q} \cdot \boldsymbol{Q}^{H}=\boldsymbol{I}\right.$, $\boldsymbol{Z} \cdot \boldsymbol{Z}^{H}=\boldsymbol{I}$ ) such that:

$$
\boldsymbol{Q} \tilde{\boldsymbol{H}} \boldsymbol{Z}^{H}=\hat{\boldsymbol{H}}, \boldsymbol{Q} \tilde{\boldsymbol{A}} \boldsymbol{Z}^{H}=\hat{\boldsymbol{A}}
$$

where both $\hat{\boldsymbol{H}}$ and $\hat{\boldsymbol{A}}$ are upper triangular matrices.

Left-multiplying (22) by $\boldsymbol{Q}$ we have:

$$
\lambda \boldsymbol{Q} \tilde{\boldsymbol{H}}\left(\boldsymbol{Z}^{H} \boldsymbol{Z}\right) \boldsymbol{T}=\boldsymbol{Q} \tilde{\boldsymbol{A}}\left(\boldsymbol{Z}^{H} \boldsymbol{Z}\right) \boldsymbol{T}
$$

According to (23), equation (24) will be (define $\boldsymbol{T}^{\prime}=\boldsymbol{Z T}$ ):

$$
\lambda \hat{\boldsymbol{H}} \boldsymbol{T}^{\prime}=\hat{\boldsymbol{A}} \boldsymbol{T}^{\prime}
$$

Equation (25) is equivalent to solve homogeneous linear equations $(\lambda \hat{\boldsymbol{H}}-\hat{\boldsymbol{A}}) \boldsymbol{T}^{\prime}=\boldsymbol{0}$. The sufficient and necessary condition for the existence of non-zero $\boldsymbol{T}^{\prime}$ is: 


$$
\operatorname{det}(\lambda \hat{\boldsymbol{H}}-\hat{\boldsymbol{A}})=0
$$

Since both $\hat{\boldsymbol{H}}$ and $\hat{\boldsymbol{A}}$ are upper triangular matrices, (26) can be written as:

$$
\operatorname{det}(\lambda \hat{\boldsymbol{H}}-\hat{\boldsymbol{A}})=\prod_{i=1}^{l}\left(\lambda \hat{H}_{i i}-\hat{A}_{i i}\right)=0
$$

where $\hat{H}_{i i}$ and $\hat{A}_{i i}$ : the $i^{\text {th }}$ diagonal element of $\hat{\boldsymbol{H}}$ and $\hat{\boldsymbol{A}}$ respectively.

Therefore $\lambda$ can be solved easily as:

$$
\lambda_{i}=\hat{A}_{i i} / \hat{H}_{i i} \quad\left(\text { if } \hat{H}_{i i} \neq 0\right)
$$

Since $\tilde{\boldsymbol{H}}$ is rank deficient for our case, we can assume $\tilde{\boldsymbol{H}}$ in the format of:

$$
\tilde{\boldsymbol{H}}=\left[\begin{array}{ll}
\boldsymbol{I}_{n \times n} & \boldsymbol{0}_{n \times m} \\
\boldsymbol{O}_{m \times n} & \boldsymbol{\varepsilon}_{m \times m}
\end{array}\right]
$$

where $\varepsilon=\operatorname{diag}\left(\varepsilon_{1} \ldots \varepsilon_{i} \ldots \varepsilon_{m}\right) ; \varepsilon_{i} \approx 0(i=1, \ldots, m)$ : infinitesimal time constant in nature.

Then after Schur decomposition, there will be some diagonal elements $\hat{H}_{i i}$ of $\hat{\boldsymbol{H}}$ with extremely small values, which correspond to $\mathcal{E}_{i}$ in $\widetilde{\boldsymbol{H}}$ and lead to infinite eigenvalue $\lambda_{i}$ in (27). This can easily be identified as "fictitious" eigenvalues and spurned.

After eigenvalue $\lambda_{i}(i=1, \ldots, n)$ calculation, its corresponding generalized right (left) eigenvector $\widetilde{\boldsymbol{e}}_{i}\left(\tilde{\boldsymbol{f}}_{i}\right)$ can be calculated by:

$$
\lambda_{i} \tilde{\boldsymbol{H}} \widetilde{\boldsymbol{e}}_{i}=\tilde{\boldsymbol{A}} \widetilde{\boldsymbol{e}}_{i}, \lambda_{i} \tilde{\boldsymbol{H}} \tilde{\boldsymbol{f}}_{i}=\tilde{\boldsymbol{A}}^{\mathrm{T}} \tilde{\boldsymbol{f}}_{\boldsymbol{i}}
$$

where $\tilde{\boldsymbol{e}}_{i}=\left[\begin{array}{c}\boldsymbol{e}_{i} \\ \boldsymbol{e}^{\prime}{ }_{i}\end{array}\right], \tilde{\boldsymbol{f}}_{i}=\left[\begin{array}{c}\boldsymbol{f}_{i} \\ \boldsymbol{f}^{\prime}{ }_{i}\end{array}\right]\left(\left(\boldsymbol{e}_{i}, \boldsymbol{f}_{i}\right)\right.$ is defined in (20)) $)$. It is easy to derive:

$$
\boldsymbol{e}_{i}^{\prime}=-\boldsymbol{D}^{-1} \boldsymbol{C} \boldsymbol{e}_{i} ;, \boldsymbol{f}_{i}^{\prime}=-\left(\boldsymbol{B} \boldsymbol{D}^{-1}\right)^{\mathrm{T}} \boldsymbol{f}_{i}
$$

It is well known that $\left(\boldsymbol{e}_{i}, \boldsymbol{f}_{i}\right)$ provides the information of observability and controllability of state variables $\boldsymbol{X}$ on mode $\lambda_{i}$, while $\left(\boldsymbol{e}_{i}^{\prime}, \boldsymbol{f}_{i}^{\prime}\right)$ provides the similar information of algebraic variables $\boldsymbol{Y}$ on mode $\lambda_{i}$. The information is very useful for designing controllers for SSO damping. In this paper, the generalized eigenvectors are normalized such that (29) is satisfied:

$$
\tilde{\boldsymbol{f}}_{i}^{\mathrm{T}} \tilde{\boldsymbol{H}} \widetilde{\boldsymbol{e}}_{i}=1
$$

\section{B. Eigenvalue Sensitivity Analysis Based on LDAE Model}

The formula for generalized eigenvalue sensitivity analysis is derived below.

Differentiating $\tilde{\boldsymbol{A}} \tilde{\boldsymbol{e}}_{i}=\lambda_{i} \tilde{\boldsymbol{H}} \tilde{\boldsymbol{e}}_{i}$ with respect to a certain parameter $K$ yields $(\because \partial \tilde{\boldsymbol{H}} / \partial K=\boldsymbol{0})$ :

$$
\frac{\partial \tilde{\boldsymbol{A}}}{\partial K} \widetilde{\boldsymbol{e}}_{i}+\tilde{\boldsymbol{A}} \frac{\partial \tilde{\boldsymbol{e}}_{i}}{\partial K}=\frac{\partial \lambda_{i}}{\partial K} \tilde{\boldsymbol{H}} \widetilde{\boldsymbol{e}}_{i}+\lambda_{i} \tilde{\boldsymbol{H}} \frac{\partial \tilde{\boldsymbol{e}}_{i}}{\partial K}
$$

Multiplying (30) by $\tilde{\boldsymbol{f}}_{i}^{T}$ from the left gives:

$$
\tilde{\boldsymbol{f}}_{i}^{T} \frac{\partial \tilde{\boldsymbol{A}}}{\partial K} \tilde{\boldsymbol{e}}_{i}+\tilde{\boldsymbol{f}}_{i}^{T} \tilde{\boldsymbol{A}} \frac{\partial \tilde{\boldsymbol{e}}_{i}}{\partial K}=\frac{\partial \lambda_{i}}{\partial K} \tilde{\boldsymbol{f}}_{i}^{T} \tilde{\boldsymbol{H}} \tilde{\boldsymbol{e}}_{i}+\lambda_{i} \tilde{\boldsymbol{f}}_{i}^{T} \tilde{\boldsymbol{H}} \frac{\partial \tilde{\boldsymbol{e}}_{i}}{\partial K}
$$

From (29) we can see the first item on the right hand side of (31) is actually $\partial \lambda_{i} / \partial K$; and the last items on both sides of
(31) are equal and hence can be eliminated. Finally we can reach:

$$
\frac{\partial \lambda_{i}}{\partial K}=\tilde{\boldsymbol{f}}_{i}^{T} \frac{\partial \tilde{\boldsymbol{A}}}{\partial K} \tilde{\boldsymbol{e}}_{i}
$$

There are only a few elements in $\tilde{A}$ with parameter $K$ explicitly existing, so $\partial \tilde{A} / \partial K$ is extremely sparse which makes eigenvalue sensitivity analysis very efficient based on the LDAE model. When $K$ exists in $\boldsymbol{D}$, the advantage of (32) is apparent.

\section{COMPUTER STUDY RESUlTS}

The IEEE first benchmark model [8] is taken as the test system to conduct eigen-analysis using proposed LDAE model and methods. The test results are presented below.

The studied system is shown schematically in figure 1 and the detailed system parameters are referred from [8]. For the convenience of modular construction of the LDAE model, we add a fictitious shunt capacitance on each bus except generator bus, and its value is set at 0.01 (p.u.). Mechanical damping for each spring mass is supposed to be 0.01 (p.u.) with mutual damping between adjacent masses neglected.

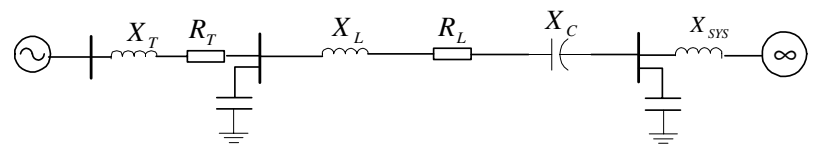

Figure 1 The first benchmark model network

The eigenvalue computation results based on LDAE model via generalized Schur decomposition method are completely the same as that of using traditional eigenvalue computation methods. Since the limit of space, only eigenvalue sensitivity analysis results are given in this paper.

Series compensation capacitor's effect on SSO is tested at first. The results are shown in figure 2. Figures 2 (a) \& (c) give the real part \& imaginary part of torsional mode $3\left(\lambda_{3}\right)$ calculated through generalized eigen-analysis under different series compensation level $k_{C}(\%)$. From figure 2 (a) we can see that the real part of $\lambda_{3}$ is positive in certain compensation range that means torsional mode 3 is unstable in this compensation range. Figure 2 (c) shows that the imaginary part of $\lambda_{3}\left(\operatorname{Im}\left(\lambda_{3}\right)\right.$ : oscillation frequency) only varies with the change of $k_{C}$ slightly and $\Delta \operatorname{Im}\left(\lambda_{3}\right)$ is relatively bigger when torsional mode 3 is unstable, but the maximum shift is less than $0.5 \%$ of the natural frequency of torsional mode 3 . We can calculate the derivative of the real \& imaginary part of $\lambda_{3}$ w.r.t. $k_{C}$ based on figure 2 (a) \& (c), which is actually the slope of curves in figures 2 (a) \& (c). And we can also use equation (32) to compute the eigenvalue $\lambda_{3}$ sensitivity w.r.t. $k_{C}$ directly. The real \& imaginary parts of $\partial \lambda_{3} / \partial k_{C}$ w.r.t. different compensation level $k_{C}$ are also plotted and shown in figures 2 (b) \& (d). Obviously, the slope of curve in figure 2 (a) is compared favorably with figure 2 (b), and the slope of curve in figure 2 (c) is consistent very well with figure 2 (d), which shows clearly the effectiveness of generalized eigenvalue sensitivity analysis based on the LDAE model.

At the series compensation level $\left(k_{\mathrm{C}}=58 \%\right)$ where mode $\lambda_{3}$ is strongly stimulated, intensive sensitivity studies of $\lambda_{3}$ w.r.t 
the change of line resistance $R_{\mathrm{L}}$, exciter gain $K_{\mathrm{E}}$ and bus fictitious shunt capacitance $C_{\text {shunt }}$ are conducted. The computer test results are listed in tables 1, 2 and 3 respectively. From table 1 we can see that the real part of $\lambda_{3}$ decreases when increasing $R_{\mathrm{L}}$, and torsional mode 3 gradually tends to be stable. Table 2 shows that the exciter gain $K_{\mathrm{E}}$ has marginal effect on torsional mode $\lambda_{3}$ for the tested case. From table 3 we can find that adding small fictitious shunt capacitance on buses will bring about neglectable effects on system torsional oscillation characteristics. However, it can significantly speed up the modularized construction of LDAE model when a bus has no charging or shunt capacitance on it.

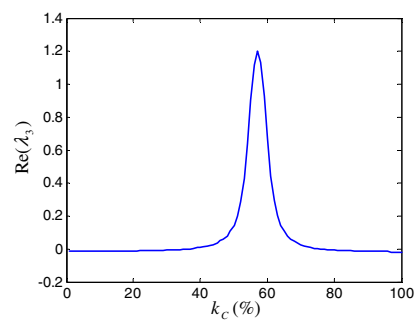

(a) $\operatorname{Re}\left(\lambda_{3}\right)$

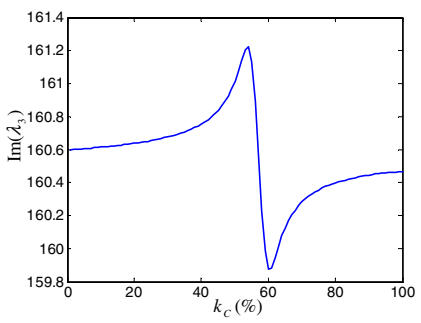

(c) $\operatorname{Im}\left(\lambda_{3}\right)$

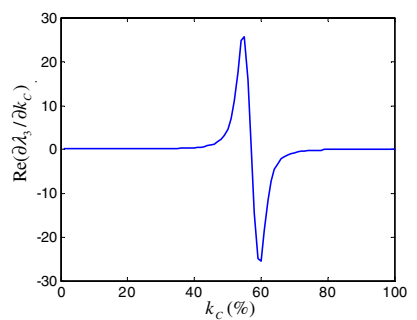

(b) $\operatorname{Re}\left(\partial \lambda_{3} / \partial k_{C}\right)$

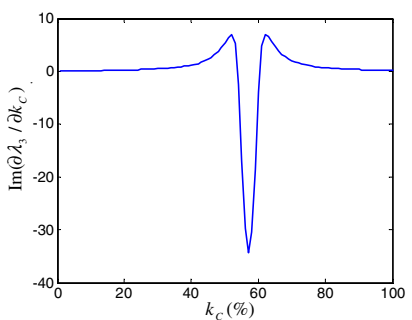

(d) $\operatorname{Im}\left(\partial \lambda_{3} / \partial k_{C}\right)$
Figure 2 Eigenvalue sensitivity analysis for torsional mode 3 with the change of series compensation

Table I

$\partial \lambda_{3} / \partial R_{\mathrm{L}}$ evaluation results $\left(\lambda_{3}\right.$ in $\left.1 / \mathrm{sec}\right)$

\begin{tabular}{ccccc}
\multicolumn{5}{c}{$\partial \lambda_{3} / \partial R_{\mathrm{L}}$ evaluation results $\left(\lambda_{3}\right.$ in $\left.1 / \mathrm{sec}\right)$} \\
\hline$R_{\mathrm{L}}(\mathrm{pu})$ & $\operatorname{Re}\left(\lambda_{3}\right)$ & $\operatorname{Im}\left(\lambda_{3}\right)$ & $\operatorname{Re}\left(\partial \lambda_{3} / \partial R_{\mathrm{L}}\right)$ & $\operatorname{Im}\left(\partial \lambda_{3} / \partial R_{\mathrm{L}}\right)$ \\
\hline 0.01 & 1.5765 & 159.9654 & -60.801 & 32.495 \\
0.02 & 1.1121 & 160.2142 & -34.144 & 17.632 \\
0.03 & 0.8384 & 160.3472 & -21.090 & 9.337 \\
0.04 & 0.6626 & 160.4202 & -14.019 & 5.225 \\
\hline
\end{tabular}

Table II

$\partial \lambda_{3} / \partial K_{\mathrm{E}}$ evaluation results $\left(\lambda_{3}\right.$ in $\left.1 / \mathrm{sec}\right)$

\begin{tabular}{lcccc}
\multicolumn{5}{c}{$\partial \lambda_{3} / \partial K_{\mathrm{E}}$ evaluation results $\left(\lambda_{3}\right.$ in $\left.1 / \mathrm{sec}\right)$} \\
\hline$K_{\mathrm{E}}(\mathrm{pu})$ & $\operatorname{Re}\left(\lambda_{3}\right)$ & $\operatorname{Im}\left(\lambda_{3}\right)$ & $\operatorname{Re}\left(\partial \lambda_{3} / \partial K_{\mathrm{E}}\right)$ & $\operatorname{Im}\left(\partial \lambda_{3} / \partial K_{\mathrm{E}}\right)$ \\
\hline 50 & 1.1121 & 160.2141 & $3.3943 \mathrm{e}-6$ & $-2.3526 \mathrm{e}-6$ \\
200 & 1.1126 & 160.2138 & $3.3983 \mathrm{e}-6$ & $-2.3591 \mathrm{e}-6$ \\
\hline
\end{tabular}

Table III

\begin{tabular}{ccc}
\multicolumn{2}{c}{$\partial \lambda_{3} / \partial C_{\text {shunt }}$} & evaluation results \\
\hline$C_{\text {shunt }}(\mathrm{pu})$ & $\operatorname{Re}\left(\lambda_{3}\right)$ & $\operatorname{Im}\left(\lambda_{3}\right)$ \\
\hline 0.01 & 1.0989 & 160.1912 \\
0.05 & 1.1121 & 160.2141 \\
\hline
\end{tabular}

\section{CONCLUSION}

In this paper, a novel structure-preserving LDAE model for power system SSO study is derived. The most significant feature of LDAE-based SSO model is the fast and systematic modularized formulation of bulk system model with meaningful system parameters and structures preserved. Generalized eigen-analysis technique using generalized Schur decomposition can be directly applied to the LDAE model for
SSO. The results can provide the information of observability and controllability of algebraic variables on individual SSO modes. Using LDAE model is also very convenient to conduct eigenvalue sensitivity analysis with extended system matrix and generalized right $\&$ left eigenvectors. The computer test results show clearly the effectiveness of the proposed LDAE model and the relevant generalized eigen-analysis method for SSO. The LDAE-based SSO study model paves the way to include HVDC transmission and FACTS device models in SSO study and get deep insight about their effects on SSO.

\section{ACKNOWLEDGMENT}

The research is supported by NSF of China (No. 50337010), National Key Basic Research Special Fund, P.R. China (2004CB217900), and the RGC Central Allocation Group Research project (CityU 01/C), Hong Kong Government, to whom the authors would like to express their sincere acknowledgements.

\section{REFERENCES}

[1] IEEE Committee. "The $4^{\text {th }}$ Supplement to a Bibliography for SSR", IEEE Trans. On PWRS, 1997

[2] N.Rostamkolai, R.J.Piwko, et al. Subsynchronous Torsional Interactions with Static VAR Compensators Influence of HVDC. IEEE Trans. On Power Systems, 1991, 6(1):255 - 261.

[3] Pilotto, L.A.S.; Bianco, A.; Long, W.F.; Edris, A.-A.; Impact of TCSC Control Methodologies on Subsynchronous Oscillations. IEEE Trans. On Power Delivery, 2003, 18(1):243 - 252

[4] Wan Bo, Zhang Yan, "Damping subsynchronous oscillation using UPFC-a FACTS device", Power System Technology, 2002. Proceedings. PowerCon 2002. International Conference on , Volume: 4 , 13-17 Oct. 2002 Pages:2298 - 2301 vol. 4

[5] IEEE Committee. "Reader's Guide to Subsynchronous Resonance", IEEE Trans. On Power Systems, 1992, 7(1):150 - 157

[6] IEEE Committee Report, "Comparison of SSR Calculations and Test Results", IEEE Trans. On Power Systems, Vol. 4, No. 1, pp. 336-344, February 1989.7

[7] Canay I. M., "A Novel Approach to the Torsional Interactions and Electrical Damping of the Synchronous Machine, Part-I: Theory, Part-II: Application to an arbitrary network", IEEE Transactions on Power Apparatus and Systems, Vol. PAS-101, No.10, pp. 3630-3640, 1982

[8] IEEE SSR Working Group. "First Benchmark Model for Computer Simulation of SSR", IEEE Trans. On PAS, 1977, 96(5): 1565-1572

[9] P. M. Anderson, B. L. Agrawal, J. E. Van Ness. Subsynchronous Resonance in Power Systems, IEEE Press, New York, 1990

[10] Anderson, E., Z. Bai, C. Bischof, S. Blackford, J. Demmel, J. Dongarra, J. Du Croz, A. Greenbaum, S. Hammarling, A. Mckenney, and D. Sorensen, "LAPACK User's Guide", Third Edition, SIAM, Philadelphia, 1999

\section{BIOGRAPHIES}

Chang Yu received the B.S. and M.S. degrees in electrical engineering from Huazhong University of Science and Technology (HUST) in 1999 and 2001, respectively. Now he is a PHD candidate in EEE Dept., Hong Kong University. His current research interest is the analysis of power system subsynchronous oscillation.

Yixin Ni (SM'94) received her B. Eng., M. Eng., and Dr. Eng. degrees all in electrical engineering, from Tsinghua University, China. She was a former Professor and Director of National Power System Lab, Tsinghua University and now is with the University of Hong Kong. Her interests are power system stability and control, FACTS, AI applications in power systems and power markets. 